\title{
Poor efficacy of the phosphorylated high-molecular-weight neurofilament heavy subunit serum level, a biomarker of axonal damage, as a marker of chemotherapy-induced peripheral neuropathy
}

\author{
MASAHIKO SUMITANI ${ }^{1}$, TORU OGATA $^{2}$, AKINA NATORI $^{3}$, JUN HOZUMI $^{1}$, NOBUTAKE SHIMOJO $^{4}$, \\ KUMIKO KIDA $^{3}$, HIDEKO YAMAUCHI ${ }^{3}$ and TERUO YAMAUCHI ${ }^{3}$ \\ ${ }^{1}$ Department of Pain and Palliative Medicine, The University of Tokyo Hospital, Tokyo 113-0033; \\ ${ }^{2}$ Department of Rehabilitation for the Movement Functions, Research Institute, National Rehabilitation Center \\ for Persons with Disabilities, Tokorozawa, Saitama 359-8555; ${ }^{3}$ The Oncology and Breast Centers, \\ St. Luke's International Hospital, Tokyo 104-8560; ${ }^{4}$ Department of Critical Care Medicine, \\ Tsukuba University Hospital, Tsukuba, Ibaraki 305-8576, Japan
}

Received January 18, 2016; Accepted April 4, 2016

DOI: 10.3892/br.2016.648

\begin{abstract}
The phosphorylated form of the high-molecular-weight neurofilament heavy subunit (pNF-H) is a major structural protein in axons. The pNF-H level is elevated in the serum of certain patients with central nervous disorders, including chemotherapy-induced cognitive impairment. The present study was conducted to elucidate the potential role of pNF-H as a marker of chemotherapy-induced peripheral neuropathy (CIPN). A total of 71 patients with early breast cancer in various stages of treatment (following 1, 3 or 7 cycles of chemotherapy, or a previous history of breast cancer chemotherapy) were assessed with a self-administered PainDETECT questionnaire [pain location, pain intensity on an 11-point numeric rating scale (NRS), and various pain qualities] and a single serum pNF-H measurement. Patients were divided into two groups based on the presence or absence of bilateral symmetric pain in the distal portions of the extremities [CIPN(+) or CIPN(-)]. The $\chi^{2}$ and Mann-Whitney tests were used for statistical analyses. Among the participants, only 8 patients complained of CIPN. Their pain intensity
\end{abstract}

Correspondence to: Dr Masahiko Sumitani, Department of Pain and Palliative Medicine, The University of Tokyo Hospital, 7-3-1 Hongo, Tokyo 113-0033, Japan

E-mail: sumitanim-ane@h.u-tokyo.ac.jp

Abbreviations: CIPN, chemotherapy-induced peripheral neuropathy; $\mathrm{NRS}$, numeric rating scale; pNF-H, phosphorylated form of the high-molecular-weight neurofilament subunit

Key words: chemotherapy, peripheral neuropathy, phosphorylated high-molecular-weight neurofilament heavy subunit, biomarker was $3.5 \pm 1.9$ (mean \pm standard deviation) compared with $1.5 \pm 1.8$ in the CIPN(-) group $(\mathrm{P}<0.01)$. The NRS of numbness in the $\mathrm{CIPN}(+)$ group was significantly higher $(2.4 \pm 1.4)$ than that of the CIPN(-) group (1.0 \pm 1.0$)$. Increased pNF-H levels were observed in $37.5 \%$ of the CIPN(+) patients and in $23.8 \%$ of CIPN(-) patients $(\mathrm{P}=0.40)$. In conclusion, CIPN is observed in the most distal portions of the peripheral nerves that are composed of dendrites but not axons. Although serum pNF-H is a biomarker of axonal damage, it is not useful as a marker of CIPN.

\section{Introduction}

Pain is a frequent symptom among cancer patients, with $30 \%$ of patients experiencing pain at the time of diagnosis and $<90 \%$ of patients experiencing pain in the advanced stages of the disease (1). The two most common etiologies of cancer pain are pain from the cancer itself or pain as a side effect of the treatments that patients have received. The pain experienced by cancer patients is usually mixed, with nociceptive and neuropathic components. Among patients with neuropathic cancer pain, $69 \%$ of them experienced tumor-related pain and $<43 \%$ experienced treatment-related pain (2). In the treatment-related pain patients, $79 \%$ of cases were due to chemotherapy or biological therapy. Nociceptive pain is usually responsive to non-steroidal anti-inflammatory drugs and opioids, whereas neuropathic pain is persistent and frequently refractory to various pharmacotherapies including opioids. In particular, chemotherapy-induced peripheral neuropathy (CIPN) can lead not only to neuropathic pain, but also to the impairment of fine motor skills and the ability to walk, profoundly affecting activities of daily living and health-related quality of life in patients with CIPN. Recent advances in cancer therapy offer improved survival for cancer patients, and the number of long-term cancer survivors is increasing, which makes CIPN a much more serious problem in cancer patients. As CIPN is 
an essentially subjective phenomenon, the lack of sensitive and objective biomarkers hinders the early diagnosis of CIPN and limits the opportunities to discontinue or alter therapy prior to the development of irreversible nerve damage.

The phosphorylated form of the high molecular weight neurofilament heavy subunit ( $\mathrm{pNF}-\mathrm{H})$ is a major structural protein present in central and peripheral axons. The pNF-H level is elevated in the serum of patients with spinal cord injury; the level of elevation reflects the severity of spinal cord injury and may serve as a biomarker of treatment efficacy in patients with spinal cord injury $(3,4)$. Additionally, our previous study (5) reported that serum pNF-H levels in patients undergoing chemotherapy for breast cancer increased in a cumulative dose-dependent manner, suggesting its potential application as a biomarker of neural damage following chemotherapy. The neural damage assessed in that study was chemotherapy-induced cognitive impairment, characterized by cognitive dysfunction, such as deficits in attention, concentration, executive function, verbal or visual learning, and processing speed in patients undergoing chemotherapy (6). However, pNF-H is certainly included in the peripheral nerve axon and an animal study reported that oxaliplatin can lead to loss of pNF-H immunoreactivity in the dorsal root ganglion (7). Therefore, the present study was conducted to elucidate the potential role of serum $\mathrm{pNF}-\mathrm{H}$ as a marker of CIPN.

\section{Materials and methods}

Patients. The present study was an ancillary analysis to our previous study (5), and the characteristics of the patients were as described previously. Briefly, 71 patients exhibited histological proof of early breast cancer and were at various stages of receiving chemotherapy (such as had received 1, 3 or 7 cycles of their regimen, and survivors who had previously completed chemotherapy). Serum pNF-H levels were measured at the time of enrollment into the study. The serum pNF-H level was determined with a commercially available enzyme-linked immunosorbent assay kit (Human Phosphorylated Neurofilament H ELISA; BioVendor, Modrice, Czech Republic), following the manufacturer's protocol. Serum samples were diluted 3-fold prior to the analysis. Serum pNF-H levels of $>70.5 \mathrm{pg} / \mathrm{ml}$ were considered positive (3). The patients were assessed by a self-administered PainDETECT questionnaire (Japanese version), which is a screening questionnaire used to identify peripheral neuropathy and neuropathic pain $(8,9)$. The PainDETECT measures pain intensity on an 11-point numerical rating scale, localizes the pain with a pain drawing, and quantifies seven types of pain using a 6-point numerical rating scale (for example: $0=$ never, $5=$ very strongly). The pain drawing contained a gender-neutral anterior and posterior outline view of a body. When the patient marked pain areas on the distal ends of four limbs, the patient was considered to exhibit CIPN. The patients were divided into two groups based on the presence or absence of CIPN.

Statistical analyses. All the statistical analyses were performed using SPSS software (IBM Corp., Armonk, NY, USA). The $\chi^{2}$ and Mann-Whitney tests were used to compare the data.
Table I. Clinical features of patients with or without CIPN.

\begin{tabular}{lccc}
\hline Characteristics & CIPN(+) & CIPN(-) & P-value \\
\hline Age, years & $47.5 \pm 6.8$ & $47.9 \pm 9.8$ & 0.941 \\
Total, no. (pNF-H positive) & $8(3)$ & $63(15)$ & $0.397^{\mathrm{a}}$ \\
Completed chemotherapy & & & \\
cycles, no. (pNF-H positive) & & & \\
1 & $1(0)$ & $19(1)$ & \\
3 & $0(0)$ & $20(6)$ & \\
7 & $5(3)$ & $14(8)$ & \\
Previously completed & $2(0)$ & $10(0)$ & \\
chemotherapy & & & \\
Pain intensity, no. & $3.5 \pm 1.9$ & $1.5 \pm 1.8$ & 0.003 \\
PainDETECT, no. & & & \\
Burning & $1.1 \pm 0.6$ & $1.0 \pm 1.0$ & 0.346 \\
Tingling and pricking & $2.5 \pm 0.9$ & $1.3 \pm 1.4$ & 0.012 \\
Tactile allodynia & $1.8 \pm 0.7$ & $1.2 \pm 1.2$ & 0.050 \\
Electric shock-like & $1.4 \pm 0.5$ & $1.0 \pm 0.9$ & 0.086 \\
Thermal allodynia & $1.6 \pm 1.2$ & $1.0 \pm 1.1$ & 0.127 \\
Numbness & $2.4 \pm 1.4$ & $1.0 \pm 1.0$ & 0.006 \\
Hyperpathia & $2.9 \pm 1.1$ & $1.4 \pm 1.4$ & 0.007 \\
Serum pNF-H, pg/ml & $428 \pm 951$ & $195 \pm 783$ & 0.262 \\
\hline
\end{tabular}

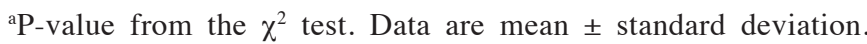
CIPN, chemotherapy-induced peripheral neuropathy; pNF-H, phosphorylated form of the high-molecular-weight neurofilament subunit.

$\mathrm{P}<0.05$ was considered to indicate a statistically significant difference.

Ethical approval. Written informed consent was obtained from all the participants, and the study was approved by the institutional review board.

\section{Results}

Patient variables. A total of 71 patients were eligible for this study. Twenty patients had completed 1 cycle of chemotherapy, 20 had completed 3 cycles, 19 had completed 7 cycles, and 12 had completed chemotherapy $\geq 24$ months prior to this study. The patient demographic data are summarized in our previous study (5). Eight patients were judged as CIPN positive [CIPN(+) group]; 1 had completed 1 cycle, 5 had completed 7 cycles, and 2 had a history of previously completed chemotherapy (Table I). The other 63 participants complained of pain in the lower lumbar area chiefly, and were categorized into the CIPN negative [CIPN(-) group]. The pain intensity of the CIPN(+) group $(3.5 \pm 1.9$; mean \pm standard deviation) was significantly higher than that of the CIPN(-) group $(1.5 \pm 1.8, \mathrm{P}<0.01)$. Regarding pain quality, tingling or pricking sensation, numbness, and hyperpathia reached statistical significance; however, a burning sensation, tactile allodynia, electric shock-like and thermal allodynia were not significant (Table I). Increased pNF-H levels were observed 
in $37.5 \%$ of the CIPN(+) patients and in $23.8 \%$ of the CIPN(-) patients $(\mathrm{P}=0.40)$. The $\mathrm{pNF}-\mathrm{H}$ levels were comparable between the $\mathrm{CIPN}(+)$ and (-) groups $(\mathrm{P}=0.26)$ (Table I).

\section{Discussion}

Among the patients in the present study, only 8 demonstrated the glove-and-stocking pain pattern indicative of peripheral neuropathy. The pain in these patients was accompanied by numbness, which is a typical symptom of CIPN. Consistent with the notion that chemotherapy impairs the function of the peripheral nervous system in a cumulative dose-dependent manner (10), the majority of the CIPN(+) patients had completed 7 cycles of chemotherapy or had previously completed full cycles of chemotherapy. However, despite the presence of CIPN symptoms, CIPN(+) patients did not demonstrate elevated serum pNF-H levels compared to CIPN(-) patients. In our previous study (5), the chemotherapy-associated pNF-H positivity rate increased in a cumulative dose-dependent manner. Furthermore, as none of the patients who had completed chemotherapy $\geq 24$ months previously showed increased serum pNF-H levels, neuronal toxicity by chemotherapy would operate temporally. By contrast, some of the present patients, who had completed chemotherapy $\geq 24$ months before pNF-H measurement, exhibited the CIPN symptom. Considering the present and previous findings together, serum $\mathrm{pNF}-\mathrm{H}$ is not a useful marker of CIPN, but remains a promising biomarker of neural damage in the central nervous system following chemotherapy.

Although pNF-H is certainly present in the peripheral nerve axon and oxaliplatin can lead to a loss of pNF-H immunoreactivity in the dorsal root ganglion (7), symptoms of peripheral neuropathy with increasing serum pNF-H levels were not observed in the present patients, as in serum pNF-H-negative patients. During the progression of CIPN, the degeneration of small unmyelinated $\mathrm{C}$ fibers usually antecedes the degeneration of thick myelinated fibers. As pNF-H is a major structural protein in the peripheral axons, the volume of $\mathrm{C}$ fibers may be too small to produce detectable serum pNF-H levels. While pNF-H is present in the peripheral axon, the most distal portions of the peripheral nerve fibers that are damaged by chemotherapy are composed of dendrites, not axons. It is not clear whether pNF-H is present in dendrites as well as axons. This may explain the present finding that CIPN(+) patients do not necessarily exhibit increased serum pNF-H levels in proportion to the number of completed cycles of chemotherapy.

\section{Acknowledgements}

The present study was supported by the Health Labour Science Research Grants from the Japanese Ministry of Health, Labour and Welfare (nos. H24-Ganrinsho-ippan-011 and H26-Kakus hintekigan-ippan-060).

\section{References}

1. Khosravi SP, Del Castillo RA and Perez MG: Management of cancer pain. An Med Interna 24: 553-556, 2007.

2. García de Paredes ML, del Moral GonzálezF, Martínez del PradoP, Martí Ciriquián JL, Enrech Francés S, Cobo Dols M, Esteban González E, Ortega Granados AL, Majem Tarruella M, Cumplido Burón JD, et al: First evidence of oncologic neuropathic pain prevalence after screening 8615 cancer patients. Results of the On study. Ann Oncol 22: 924-930, 2011.

3. Hayakawa K, Okazaki R, Ishii K, Ueno T, Izawa N, Tanaka Y, Toyooka S, Matsuoka N, Morioka K, Ohori Y, et al: Phosphorylated neurofilament subunit NF-H as a biomarker for evaluating the severity of spinal cord injury patients, a pilot study. Spinal Cord 50: 493-496, 2012.

4. Ueno T, Ohori Y, Ito J, Hoshikawa S, Yamamoto S, Nakamura K, Tanaka S, Akai M, Tobimatsu Y and Ogata T: Hyperphosphorylated neurofilament NF-H as a biomarker of the efficacy of minocycline therapy for spinal cord injury. Spinal Cord 49: 333-336, 2011.

5. Natori A, Ogata T, Sumitani M, Kogure T, Yamauchi $T$ and Yamauchi H: Potential role of pNF-H, a biomarker of axonal damage in the central nervous system, as a predictive marker of chemotherapy-induced cognitive impairment. Clin Cancer Res 21: 1348-1352, 2015.

6. O'Farrell E, MacKenzie J and Collins B: Clearing the air: A review of our current understanding of 'chemo fog'. Curr Oncol Rep 15: 260-269, 2013

7. Jamieson SM, Subramaniam J, Liu JJ, Jong NN, Ip V, Connor B and McKeage MJ: Oxaliplatin-induced loss of phosphorylated heavy neurofilament subunit neuronal immunoreactivity in rat DRG tissue. Mol Pain 5: 66, 2009.

8. Freynhagen R, Baron R, Gockel U and Tölle TR: painDETECT: A new screening questionnaire to identify neuropathic components in patients with back pain. Curr Med Res Opin 22: 1911-1920, 2006.

9. Matsubayashi Y, Takeshita K, Sumitani M, Oshima Y, Tonosu J, Kato S, Ohya J, Oichi T, Okamoto N and Tanaka S: Validity and reliability of the Japanese version of the painDETECT questionnaire: A multicenter observational study. PLoS One 8: e68013, 2013.

10. Cavaletti G, Cornblath DR, Merkies IS, Postma TJ, Rossi E, Frigeni B, Alberti P, Bruna J, Velasco R, Argyriou AA, et al; CI-PeriNomS Group: The chemotherapy-induced peripheral neuropathy outcome measures standardization study: From consensus to the first validity and reliability findings. Ann Oncol 24: 454-462, 2013. 